\section{A inclusão digital de pessoas idosas em momento de pandemia: relato de experiência de um projeto de extensão}

\author{
The digital inclusion of older people in a
andemic: experience report from an extension \\ The digital inclusion of older people in a
pandemic: experience report from an extension \\ project
}

Tainá Maria Silva Deodoro ${ }^{1}$, Lilian Dias Bernardo ${ }^{2}$, Allana Karoline Chaves da Silva ${ }^{3}$, Taiuani Marquine Raymundo ${ }^{4}$, Isabela Vinharski Scheidt ${ }^{5}$
Revista

Extensão em Foco

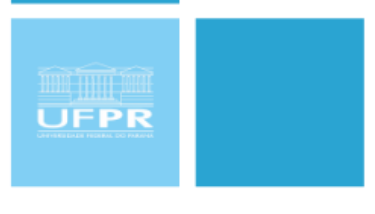

ISSN $2358-7180$

\title{
RESUMO
}

Objetivo: apresentar o desenvolvimento de um projeto de inclusão digital para idosos realizado em um ambiente virtual de aprendizagem. Métodos: Relato de experiência sobre o desenvolvimento de um projeto remoto de inclusão digital para idosos ofertado em Curitiba e Rio de Janeiro. Os participantes foram convidados por meio de grupos no WhatsApp ${ }^{\circledR}$ e divulgação nas redes sociais. Os critérios para participação foram: ter 60 anos ou mais, possuir Smartphone com acesso à internet, capacidade para baixar o aplicativo Google Meet e habilidade para enviar mensagens pelo

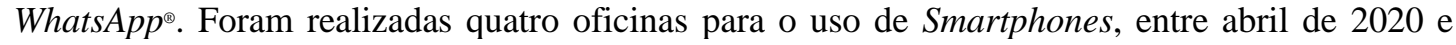
janeiro de 2021, duas em Curitiba e duas no Rio de Janeiro. Os encontros foram conduzidos por 19 estudantes do curso de Terapia Ocupacional. Resultado: Participaram das oficinas 47 idosos, com idades entre 61 e 85 anos, a maioria do sexo feminino. $\mathrm{Na}$ análise dos relatos, as barreiras indicadas pelas monitoras que conduziram as oficinas estiveram relacionadas à própria tecnologia, ao ambiente, ao processo de ensino e aprendizagem e decorrentes de fatores pessoais. Como facilitadores, destacaram-se a confecção do material instrucional em vídeo, a troca da plataforma de ensino, a possibilidade de reagendamento dos encontros e a divisão de tarefas em duplas. Conclusão: Com o contexto da pandemia, o projeto de inclusão digital ocorreu no formato virtual e evidenciou a importância enquanto prática para promover o uso da tecnologia, o aprendizado e desenvolvimento de competências e, principalmente, favorecer a inclusão social dos idosos em momentos adversos.

Palavras-chave: Pessoa Idosa. Inclusão Digital. Ambiente Virtual de Aprendizagem.

\section{ABSTRACT}

Objective: Present the development of a digital inclusion project for older people carried out in a virtual learning environment. Methods: Experience report on the development of a remote digital inclusion project for older people offered in Curitiba and Rio de Janeiro. Participants were invited through groups on WhatsApp® and disseminated on social networks. The participation criterion was to be 60 years old or older, to have a Smartphone with internet access, the ability to download the Google Meet ${ }^{\circledR}$ application and the ability to send messages via WhatsApp®. Four workshops for the use of Smartphones

\footnotetext{
${ }^{1}$ Graduanda do curso de Terapia Ocupacional. Instituto Federal do Rio de Janeiro (IFRJ), Rio de Janeiro, Rio de Janeiro, Brasil. E-mail: tainadeodoro@gmail.com. Orcid: https://orcid.org/0000-0003-0469-3994 ${ }^{2}$ Doutora em Saúde Coletiva. nstituto Federal do Rio de Janeiro (IFRJ), Rio de Janeiro, Rio de Janeiro, Brasil. E-mail: lilian.bernardo@ifrj.edu.br. Orcid: https://orcid.org/0000-0001-5234-4225

${ }^{3}$ Graduanda do curso de Terapia Ocupacional. Universidade Federal do Paraná (UFPR), Curitiba, Paraná, Brasil. E-mail: allanakarolineh@gmail.com. Orcid: https://orcid.org/0000-0001-7368-4034

${ }^{4}$ Doutora em Ciências. Universidade Federal do Paraná (UFPR), Curitiba, Paraná, Brasil. E-mail: taiuanimarquine@gmail.com. Orcid: https://orcid.org/0000-0002-8598-463X

${ }^{5}$ Doutoranda em Educação Física. Universidade Federal do Paraná (UFPR), Curitiba, Paraná, Brasil. Email: isabelavscheidt@gmail.com. Orcid: https://orcid.org/0000-0001-9561-6120
} 
were held, from April 2020 to January 2021, two in Curitiba and two in Rio de Janeiro. The meetings were conducted by 19 students from the Occupational Therapy course. Result: 47 older people, aged between 61 and 85 years, participated in the workshops, most of them female. In the analysis of the reports, the barriers reported by the monitors who conducted the workshops were related to the technology itself, the environment, teaching and learning process and resulting from personal factors. The facilitators included the making of instructional material on video, the exchange of the teaching platform, the possibility of rescheduling meetings and the division of tasks in pairs. Conclusion: With the context of the pandemic, the digital inclusion project took place in a virtual format and highlighted the importance as a practice to promote the use of technology, learning and skills development and, mainly, to favor the social inclusion of the older people in adverse moments.

Keywords: Older People. Digital Inclusion. Virtual Learning Environment.

\section{INTRODUÇÃO}

$\mathrm{O}$ contexto da pandemia do coronavírus modificou rápido e significativamente a vida das pessoas, sobretudo dos idosos, uma vez que estes são considerados como um grupo de maior risco para a morbimortalidade em casos de adoecimento. A estas pessoas, foi aconselhado o distanciamento físico, ou seja, permanecerem em casa (CALLOW; CALLOW; SMITH, 2020).

$\mathrm{O}$ distanciamento físico e o auto isolamento têm sido as principais medidas para redução na transmissão do coronavírus e isto impacta na realização de atividades realizadas na comunidade ou daquelas que favorecem os contatos sociais, como as idas aos supermercados, bancos, restaurantes, centros de convivência, clubes, reuniões familiares e locais de celebração religiosa. Na pandemia, as atividades cotidianas têm se restringido mais ao ambiente doméstico e impõem às pessoas a criação de novas estratégias para continuar a realizar as atividades que são obrigatórias ou aquelas que promovem prazer e relaxamento.

Neste cenário, o uso de tecnologias digitais de informação e comunicação (TDIC) desvelou ser um recurso capaz de atenuar o impacto das restrições na participação em atividades rotineiras, além de ser um meio para promover conexões sociais (SEIFERT; COTTEN; XIE, 2021).

As TDIC favorecem o gerenciamento de comunicação e participação social (AOTA, 2020), contribuem para o bem-estar físico e psicológico, auxiliam na redução do isolamento (BALL et al., 2019) e no engajamento em atividades de lazer (LARSSONLUND; NYMAN, 2020), além de promover conexões sociais e possibilitar a participação 
em videoconferências, cursos remotos, acesso a serviços de telessaúde e delivery (SEIFERT; COTTEN; XIE, 2020; XIE et al., 2021).

Porém, o grupo de pessoas idosas é considerado o mais infoexcluído se comparado às demais faixas etárias, uma vez que, ao longo de suas vidas, suas experiências foram adquiridas com tecnologias muito diferentes do ambiente tecnológico existente atualmente (BALL et al., 2019). Acresce a isso, obstáculos de ordem pessoal, contextual ou decorrente da própria tecnologia, que aumenta, ainda mais, a restrição para incluir essas tecnologias ao cotidiano da pessoa idosa (TAVARES; SOUZA, 2012). Ao considerar o "confinamento" imposto pela pandemia, a pessoa idosa pode se sentir duplamente excluída nessa sociedade dominada pela tecnologia, seja pelo distanciamento físico ou pela dificuldade em utilizar, assimilar ou compreender o dispositivo tecnológico (SEIFERT; COTTEN; XIE, 2021).

Frente aos desafios apresentados, a criação e o desenvolvimento de programas de inclusão digital para idosos tem sido reconhecida como um caminho potencial para capacitar e promover independência no uso da tecnologia, com vistas à facilitar as conexões entre pessoas, auxiliar na redução da sensação de isolamento, contribuir para o bem-estar e favorecer a participação em atividades do cotidiano e a aprendizagem ao longo da vida (AOTA, 2020; BALL et al., 2019; EYNON; MALMBERG, 2021).

Na condução desses programas, aspectos relacionados ao ambiente físico e social, ao processo de ensino e aprendizagem, a própria tecnologia, assim como os fatores pessoais da pessoa idosa devem ser considerados para atingir o sucesso na aprendizagem das novas tecnologias (ALVARENGA; YASSUDA; CACHIONI, 2019; PÁSCOA; GIL, 2019).

Diante do exposto surgem reflexões e indagações ao considerar qual a potencialidade de programas que visem instrumentalizar idosos para o uso de TDIC em um cenário em que as autoridades sanitárias recomendam permanecer em casa e evitar contatos sociais. Uma outra questão é: como desenvolver um programa de ensino e aprendizagem para o uso de TDIC, utilizando o próprio dispositivo tecnológico, de forma remota, uma vez que este é o recurso mais viável e seguro para o momento pandêmico?

A partir das questões norteadoras, o objetivo desse artigo foi apresentar o desenvolvimento de um projeto de inclusão digital para idosos realizado em um ambiente virtual de aprendizagem.

\section{MÉTODOS}


Trata-se de um relato de experiência sobre o desenvolvimento de um projeto remoto de inclusão digital para idosos ofertado em Curitiba e Rio de Janeiro, coordenado por docentes do curso de Terapia Ocupacional. O projeto de inclusão digital teve início no Paraná em 2016 e teve seu pólo aberto no Rio de Janeiro, em 2018. Atualmente já atendeu mais de 260 pessoas. O objetivo do projeto é instrumentalizar pessoas idosas para o uso independente das tecnologias que fazem parte de seus cotidianos. Aqui, será abordado o Smartphone como o dispositivo móvel utilizado neste estudo.

O projeto utiliza a abordagem centrada nas demandas e necessidades do idoso, ou seja, os participantes decidem o conteúdo que desejam aprender e, por isso, o planejamento dos encontros é conduzido de forma personalizada. O projeto, em seu formato presencial, se desenvolveu por meio de uma oficina, com encontros semanais $(\mathrm{n}=10)$ que duravam 90 minutos cada, seguidos por um momento de socialização em que os participantes faziam um lanche. Desde o início da pandemia, as oficinas foram desenvolvidas de forma remota, sendo reestruturada em relação ao número de encontros e duração, com o auxílio de ferramentas síncronas e assíncronas.

Participaram do estudo remoto as pessoas idosas com idade igual ou superior a 60 anos. No período de abril de 2020 a janeiro de 2021 foram realizadas quatro oficinas de inclusão digital para o uso de Smartphones, duas em cada cidade onde o projeto ocorreu. E em toda a proposta estiveram auxiliando 10 monitores no Paraná, e 9 monitoras no Rio de Janeiro.

O convite aos participantes foi feito por meio de grupos no aplicativo WhatsApp em que participavam pessoas idosas que já haviam realizado a inclusão digital presencialmente ou que estavam em outros projetos direcionados a esse público nestas instituições, assim como foi feita a divulgação pelas redes sociais.

Como critério de seleção para a participação, as pessoas idosas precisavam ter um Smartphone com acesso à internet e, em seu dispositivo, ter a capacidade de memória para baixar o aplicativo Google Meet ${ }^{\star}$ (exceto para a primeira oficina em cada cidade) e possuir habilidade para enviar mensagens pelo aplicativo WhatsApp ${ }^{\oplus}$. Além dos prérequisitos investigados para a participação na oficina, os participantes do projeto informavam também se possuíam uma rede de apoio no ambiente doméstico para auxiliar em possíveis obstáculos que pudessem ocorrer nos encontros e que não pudessem ser sanados de forma remota. 
Os encontros eram conduzidos por estudantes do curso de graduação em Terapia Ocupacional. Após as oficinas, as monitoras elaboraram relatos a respeito da experiência na condução do projeto de inclusão, em seu formato virtual remoto.

No que tange às considerações éticas, o projeto teve aprovação dos comitês de éticas das universidades correspondentes sob pareceres número 4.589.302, de 13 de março de 2021, e 3.824.627, 6 de fevereiro de 2020. Os participantes assinaram um termo de consentimento livre e esclarecido para a participação no projeto de inclusão digital.

\section{EM BUSCA DA INCLUSÃO DIGITAL: O RELATO DE EXPERIÊNCIA}

Participaram do projeto de inclusão digital virtual 47 pessoas, sendo 42 do sexo feminino, com idades que variaram entre 61 e 85 anos. Às pessoas idosas interessadas em aprender sobre diversas funções do Smartphone, foi feito o contato para apresentação das monitoras, averiguação da presença de suporte familiar no ambiente doméstico (como já mencionado), levantamento das demandas quanto ao uso do telefone celular e dados pessoais.

Após o levantamento das necessidades do participante, cada idoso indicava o tipo de material instrucional que gostaria de receber para ter como suporte aos estudos, seja por apostila, podcasts ou vídeos explicativos. Estes conteúdos eram enviados dois dias antes do encontro virtual síncrono, pois assim o aprendiz teria tempo hábil para estudar o material.

Os recursos didáticos foram planejados para favorecer a leitura ou a visualização no próprio aparelho celular. Desse modo, as apostilas eram desenvolvidas com fontes e tamanhos de letra apropriados, as funções eram ensinadas pelo passo a passo e de acordo com cada etapa do conteúdo escolhido. Os materiais disponibilizados em vídeo foram elaborados com o auxílio de aplicativos que capturavam os comandos na tela juntamente com a voz do narrador. Estes foram editados com inserção de setas ou imagens para facilitar as instruções que estavam sendo produzidas em cada etapa da função.

As oficinas ocorreram em dois formatos: com participantes que já haviam frequentado o projeto de inclusão digital no formato presencial (as duas primeiras oficinas, uma em cada cidade) e com pessoas idosas que não tiveram experiência prévia com este tipo de projeto (as duas oficinas subsequentes). 
A assistência ao idoso no formato assíncrono era realizada pelo envio do material instrucional, assim como era garantido a cada participante a resolução de problemas com as funções do Smartphone, via mensagens pelo WhatsApp ${ }^{\circledR}$.

Os encontros síncronos foram realizados por videoconferência no WhatsApp ${ }^{\circledR}$ (as duas primeiras oficinas, uma em Curitiba e outra no Rio de Janeiro) ou no Google Meet $^{\oplus}$ (as demais oficinas), com duração média de 30 e 60 minutos. Esses encontros buscavam sanar dúvidas e investigar a aprendizagem por meio de exercícios de fixação, assim como, trocar experiências sobre as facilidades e dificuldades que encontraram no aprendizado do conteúdo enviado durante a semana.

As primeiras oficinas, em cada cidade, foram compostas, em média, por três encontros e o objetivo foi atender três demandas de interesse de cada participante. Nas duas cidades, cada dupla de monitoras era responsável por uma pessoa idosa. As funções mais solicitadas por eles foram: aprender a liberar memória no celular, baixar e excluir aplicativos, instruções para segurança no uso do Smartphone, aplicativos para realizar vídeochamada, assim como armazenar arquivos no aplicativo Google Drive ${ }^{\bowtie}$ e funções do Facebook ${ }^{\circledR}$.

Por sua vez, nas duas últimas oficinas, houve diferença no modo de condução entre as instituições proponentes. Em Curitiba, cada estudante ficou responsável por um idoso, e no Rio de Janeiro, estruturou-se a proporção de duas estudantes para duas pessoas idosas. Nesse último caso, o critério para agrupar os idosos foi por nível de conhecimento prévio no uso dos dispositivos móveis. Em Curitiba, as monitoras realizaram os encontros conforme a primeira oficina (até 3 demandas) e, no Rio de Janeiro, foi aplicada a oficina nos moldes do modelo presencial (10 encontros).

Nessas oficinas, como o aplicativo Google Meet ${ }^{\circledast}$ não era de uso rotineiro dos idosos e nem todos eles tinham familiaridade com o uso do Smartphone, as monitoras enviaram um vídeo de instruções sobre como baixar e utilizar a ferramenta, antes de iniciar o ensino das demandas solicitadas por eles. Nessa etapa, os idosos foram instruídos a fazer download do aplicativo, ativar/desativar câmera e microfone, acessar o chat e compartilhar a apresentação de tela.

As demandas mais solicitadas pelos participantes foram: armazenar arquivos no Google Drive ${ }^{\bowtie}$, deixar a tela com a luz acesa por mais tempo, bloqueio de tela, incluir e excluir compromissos no calendário, baixar e excluir aplicativos, Facebook ${ }^{\circledR}$, acessar agenda telefônica durante ligação, despertador, enviar e-mail e editar fotos. Soma-se a isso o interesse de participantes em aprender a criar uma reunião no Google Meet ${ }^{\natural}$. 
$\mathrm{Na}$ análise da condução das oficinas, barreiras relacionadas à própria tecnologia, ao ambiente, ao processo de ensino e aprendizagem ou decorrentes de fatores pessoais foram os desafios impostos para efetivar a inclusão digital.

Ao considerar o processo de ensino e aprendizagem, destaca-se as primeiras oficinas, em que se usou exclusivamente o WhatsApp ${ }^{\circledR}$ como o ambiente virtual de aprendizagem e foi percebido como obstáculo a ausência da possibilidade em compartilhar tela e, assim, não era possível ao idoso mostrar o passo a passo do que ele fazia para executar as funções que estava aprendendo. Assim, as monitoras encontraram dificuldades para acompanhar as etapas de uma função somente com as instruções faladas. Mesmo pedindo ao idoso para fazer uma captura da tela (print screen) e enviar a imagem por anexo, nem todos os participantes sabiam realizar essa função e/ou anexar foto no WhatsApp ${ }^{\oplus}$.

Ao considerar essas barreiras, optou-se pela mudança do recurso em que seriam feitos os encontros síncronos. Nesse cenário, o aplicativo Google Meet ${ }^{\circledR}$ foi escolhido por possibilitar a apresentação de tela e, consequentemente, acompanhar visualmente as etapas realizadas pelo aprendiz de forma simultânea. A troca do ambiente virtual foi considerado pelas monitoras como facilitador do processo de ensino e aprendizagem.

À semelhança, a confecção dos materiais instrucionais foi identificado como um recurso potente para agregar novos conhecimentos, conforme depoimento de uma das monitoras a seguir:

As apostilas que eu fiz para a monitoria virtual também senti que foram mais priorizadas e destacadas, do que quando fazemos presencialmente ... dão atenção para ela... virtualmente eu percebi que por se tratar especificamente das dúvidas deles, eles valorizaram mais (M. 05).

Além disso, houve relatos de que a inexperiência com a metodologia utilizada nas oficinas era um problema vivenciado pelas monitoras, especialmente nas primeiras oficinas, porque a atividade era desenvolvida no formato remoto e elas se consideravam sem expertise para conseguir acompanhar o idoso integralmente, assim como o distanciamento físico restringia a compreensão sobre as habilidades que os participantes estariam desenvolvendo. O depoimento abaixo exemplifica essa situação:

A aula, em si, se tornava mais difícil pois tiveram vezes que eu demorei mais para passar um assunto porque não conseguia ver corretamente o que o aluno estava me mostrando e o aluno não conseguia me compreender pela câmera. Isso atrapalhou bastante porque no presencial nós podemos mostrar nosso celular na frente deles para que eles acompanhem os passos e se for o caso deles "errarem" e pararem em uma tela no celular que não saibam como 
resolver (como foi o caso de uma das minhas alunas no virtual), nós podíamos mostrar no próprio celular deles como fazer (M. 05).

A insegurança, para além do formato remoto e do distanciamento físico, parecia ser atribuída ao fato de que, em muitas oficinas, não se conhecia o participante, nem seu estilo de aprendizagem. Esse formato virtual de aprendizagem era novidade para todos os envolvidos no projeto e, assim, era esperado que as pessoas se sentissem inseguras diante dos obstáculos, até então, desconhecidos. Segundo relato das monitoras, a segurança para conduzir os encontros foi surgindo à medida que os vínculos eram formados com os participantes.

Em contraponto, aparece como facilitador do processo de ensino e aprendizagem, o planejamento e desenvolvimento das oficinas em duplas, assegurando divisão de tarefas, promoção de confiança para conduzir os encontros e por reduzir o risco de que o participante não receba assistência caso ocorra problemas de ordens diversas. Uma das monitoras exemplifica a importância nesse rearranjo de trabalho em casos de instabilidade na rede de internet:

Ter trabalhado em dupla foi um dos pontos positivos, porque possibilitou dividir a confecção do material de ensino e o suporte às dúvidas e demandas dos participantes. Soma-se a isso a segurança de ter uma das monitoras presentes na condução da oficina, uma vez que, se a internet de uma apresentasse inconsistência, teria a outra à disposição, não deixando os idosos desassistidos e/ou nervosos sem saber como proceder (M. 10).

No que tange aos fatores relacionados à complexidade da tecnologia, pela diversidade dos modelos de Smartphones, a capacidade do dispositivo móvel para armazenar os recursos didáticos ou para fazer download dos materiais foram os obstáculos mais prevalentes em todas as oficinas. Isso pode ter ocorrido pela própria capacidade do dispositivo móvel ou pela quantidade de materiais (fotos, vídeos, outros aplicativos) que já estavam armazenados anteriormente pelos idosos em seus celulares. Essa barreira foi superada após a adoção do compartilhamento de material pelo Google Drive ${ }^{\circledast}$, evitando assim usar a capacidade de memória do aparelho e dando a oportunidade para que os participantes incorporassem esse novo conhecimento.

$\mathrm{Na}$ sequência, ao analisar os obstáculos decorrentes de fatores pessoais, a dificuldade para a compreensão das instruções dadas nos encontros síncronos foi uma das observações realizadas pelas monitoras. Isso era resultado do fato de que os dispositivos móveis, dependendo da marca e tipo do aparelho, conduz a explicações muito particulares, uma vez que o passo-a-passo é diferenciado entre os tipos de Smartphones. 
Esse obstáculo, na percepção das monitoras, se agravou pela dificuldade em manter o diálogo entre as monitoras e as pessoas idosas, pois os relatos indicavam pouca interação dos idosos, especialmente nos encontros iniciais. No entanto, a comunicação foi se ampliando à medida que os vínculos eram fortalecidos.

Chama a atenção os relatos sobre o baixo senso de auto eficácia e insegurança dos participantes na experimentação e vivência com seus dispositivos móveis. Uma das monitoras destaca:

Idosos com senso baixo de auto eficácia se mostravam muito hesitantes em seguir os comandos pois tinham medo de errar. Isso aconteceu mais constantemente em oficinas iniciais onde a confiança e o vínculo afetivo com a monitora ainda não estavam consolidados (M. 03).

Nesse contexto, modificações nos encontros foram adotadas de forma a melhorar o senso de auto eficácia e evidenciar a capacidade permanente de aprendizagem ao longo da vida. Para tanto, as monitoras passaram a dar feedbacks sobre as habilidades desenvolvidas (ou em desenvolvimento) em cada encontro e usavam mais de um tipo de exemplo na explicação ao sanar dúvidas sobre o material instrucional, promovendo um ambiente mais seguro para o idoso. Por outro lado, os próprios idosos buscavam fazer anotações sobre os novos conhecimentos e passaram a exercitar as funções no próprio aparelho, melhorando sua percepção sobre sua auto eficácia no manejo e para usufruir dos dispositivos móveis.

Por fim, na análise dos fatores ambientais que poderiam influenciar o processo de inclusão digital, os encontros síncronos evidenciaram entraves relacionados à instabilidade na rede de internet (tanto por parte do idoso como das monitoras), falta de tempo para estudar o conteúdo novo e reagendamento constante dos encontros. No que concerne ao ambiente social, algumas monitoras relataram a ausência de suporte familiar no decorrer das oficinas. A importância dos familiares na manutenção da motivação para a aprendizagem é um destaque observado por uma das monitoras:

\footnotetext{
Quero relatar a dificuldade com idosos que não têm suporte familiar. A falta de uma pessoa que pudesse ajudar no decorrer da semana com as tarefas, ou até mesmo nas oficinas iniciais foi muito prejudicial, já que problemas técnicos (queda da internet, lentidão do celular, ajuste de volume...) sempre ocorrem. A ajuda de uma pessoa que saiba lidar com essas situações inesperadas é essencial (M. 03).
}

No combate aos desafios encontrados, as monitoras, sempre que possível, faziam reagendamentos dos encontros para que os idosos não perdessem a oportunidade de se 
instrumentalizar para o uso de seus Smartphones. Por outro lado, não tiveram a oportunidade de adotar estratégias para conscientizar os familiares sobre o seu papel como incentivador e até mesmo mediador das atividades que favorecem a aprendizagem ao longo da vida.

Nas adversidades impostas pela pandemia, a experiência do desenvolvimento deste projeto de inclusão digital no formato remoto foi uma oportunidade dada às pessoas idosas para aprenderem como as tecnologias poderiam facilitar suas vidas, ao possibilitar realizar algumas atividades obrigatórias e prazerosas por meio do dispositivo móvel, assim como possibilitou (re)estabelecer conexões sociais de forma segura, respeitando as medidas restritivas adotadas pelas organizações de saúde.

Ao analisar o processo formativo, o desenvolvimento das oficinas permitiu que as estudantes pudessem estruturar um novo formato de ensino e aprendizagem, pensar em materiais que pudessem ser atrativos e adequados para serem estudados por meio de dispositivos móveis, assim como lidar com obstáculos antes não encontrados. Essas atividades contribuíram de forma positiva com o processo formativo das acadêmicas.

\section{DISCUSSÃO}

Diante dos elementos abordados no presente relato de experiência, torna-se relevante, primeiramente, destacar a função que o Smartphone assumiu enquanto recurso para o aprendizado sobre a tecnologia, no contexto de pandemia, embora já fosse de conhecimento as diferentes funcionalidades e aplicações desse recurso no sentido de poder fazer ligações, mandar mensagens, navegar na internet, interagir nas redes sociais e realizar reuniões em um único aparelho com numerosas funções concentradas (BORGES et al., 2016). Com a necessidade do distanciamento físico, grande parte das atividades relacionadas à educação, como aulas, eventos, cursos e oficinas tiveram que ser adequadas ao formato virtual (SANDARS et al., 2020).

Nesse cenário, o uso de dispositivos móveis aplicado às diferentes formas de ensino tornou-se evidente pela viabilidade de acesso rápido e facilidade de compartilhamento de informações (IYENGAR et al., 2020; SANDARS et al., 2020). Na realização das oficinas de inclusão digital, os Smartphones se caracterizaram como ferramenta principal para mediar o fluxo de comunicação e execução de todo o processo, abrangendo as etapas de divulgação da iniciativa, contato inicial com as monitoras, 
compartilhamento de materiais para atividades assíncronas e realização das aulas síncronas.

Nota-se, também, o papel paralelo que os dispositivos móveis ocuparam durante a aplicação das oficinas: ao mesmo tempo que os Smartphones mediaram o processo de aprendizagem, neles estavam as principais demandas e expectativas para se aprender. Algumas dúvidas se relacionaram com a própria otimização do uso do aparelho e suas funcionalidades, enquanto outras estavam relacionadas a intenção de comunicação e interação social.

A primeira situação supracitada pode ser atribuída às diferentes aplicações da tecnologia nesse momento de pandemia, como o manejo de finanças, compras, aumento do compartilhamento de arquivos, que exigiram melhor desempenho do aparelho e seu uso mais frequente (DAVID; ROBERTS, 2021). No que se refere à comunicação e interação social, nota-se que esse aspecto convergiu com a orientação de distanciamento físico. Manter o convívio social, sobretudo por meios virtuais, manifestou-se através da necessidade de se conectar com familiares, amigos, atividades culturais e religiosas, e de gerenciamento da saúde, incluindo as modalidades de tele atendimento ofertadas nesse momento (CASTRO et al., 2020; GRÁCIO, 2020; KHILNANI; SCHULZ; ROBINSON, 2020; MENDES, 2020).

Essas mudanças no padrão de oferta de informações e serviços por meios tecnológicos possibilita reflexões não somente no sentido de o idoso ter condições de acesso a esses elementos, mas, também, do quanto esses serviços podem alcançar essa população. À exemplo, nesse momento, a participação na presente proposta foi viável para pessoas idosas com Smartphone e conhecimentos básicos quanto ao seu uso, com acesso à internet e às informações de divulgação da proposta. Ainda que se reconheça esses fatores como limitação no que diz respeito a abrangência da população, o estabelecimento de tais condições como requisito para a participação foi necessária para a execução da proposta, considerando a necessidade de manter o distanciamento social.

Embora existam orientações para o planejamento e execução de projetos de inclusão digital em formatos presenciais (RAYMUNDO; GIL; BERNARDO, 2019), e também se considere a própria experiência nas oficinas de inclusão digital para pessoas idosas, o principal desafio no momento pandêmico se refere a adaptação ao contexto de trabalho remoto. Esse aspecto ficou evidente a partir dos ajustes processuais realizados tanto no decorrer das oficinas, a partir das conduções das monitoras, bem como na organização para a oferta de novas oficinas, como adequação de número de encontros, 
distribuição das monitoras e plataforma para atividades síncronas. Para realizar os devidos ajustes, constatou-se a necessidade de existir a colaboração entre todas as partes, em que principalmente o treinador - nas oficinas representadas pelas monitoras - precisa estar atento a atitude do participante, e entender as barreiras e oportunidades de cada situação de ensino e aprendizagem (RAYMUNDO; GIL; BERNARDO, 2019).

Ainda que as narrativas das monitoras tenham ocorrido nas perspectivas de identificar barreiras e facilitadores na aplicação das oficinas em situação remota, ressaltase que ambos os panoramas são permeados pelo reconhecimento de desafios. Nesse sentido, é necessário validar as estratégias de adaptação diante das condições impostas pelo momento de pandemia, e que também reforçaram a importância do projeto enquanto atividade de extensão em suas atribuições no processo de formação das alunas, bem como na aproximação das práticas das instituições de ensino às necessidades da comunidade (NUNES; SILVA, 2011).

A oferta das oficinas de inclusão digital para pessoas idosas em momento de pandemia assume um papel relevante não somente pela função atribuída à tecnologia como alternativa para reduzir o distanciamento físico, mas, principalmente, por permitir ao idoso se engajar em um processo de aprendizagem e assumir papéis ocupacionais inerentes a esse engajamento. Sabe-se que, em seu cerne, as premissas da inclusão digital apontam para a inclusão social (MARTINI, 2005). A inclusão digital promove o conhecimento e acesso a recursos que permitem ao sujeito identificar e participar de seu contexto social, proporcionando a conquista da "cidadania digital" e, como consequência, viabilizando uma sociedade mais igualitária (RAYMUNDO; GIL; BERNARDO, 2019).

O processo de aprendizagem ao longo da vida é de grande importância, e agrega à perspectiva de inclusão. Em relação à pessoa idosa, o acesso ao conhecimento e desenvolvimento de competências para o uso da tecnologia é uma forma de promover o aprendizado nessa fase da vida (MACHADO et al., 2016). Esse aspecto corrobora, portanto, com os ideais de incluir o sujeito no âmbito digital e, paralelamente, promover a participação ativa e autônoma em diferentes esferas sociais.

\section{CONSIDERAÇÕES FINAIS}

A proposta de oficinas de inclusão digital para idosos eram desenvolvidas presencialmente em momento anterior à pandemia, em duas instituições de ensino superior. No entanto, com as orientações de distanciamento físico em função da COVID- 
19, foram necessárias adequações para que a oferta continuasse de modo remoto, ainda mais em um momento em que a tecnologia se tornou um dos principais meios de comunicação, informação e acesso a serviços.

Este relato de experiência descreveu informações quanto à estrutura e organização das oficinas, sendo possível identificar os principais desafios em relação à execução das ações. Embora seja notória a necessidade de ajustar a proposta ao formato remoto, bem como considerar, individualmente, as necessidades das pessoas atendidas, a pandemia evidenciou a importância da inclusão digital de idosos enquanto prática para promover o uso da tecnologia, o aprendizado e desenvolvimento de competências e, a aprendizagem ao longo da vida e, principalmente, a inclusão social desta população.

\section{REFERÊNCIAS}

ALVARENGA, G.M.O.; YASSUDA, M.S.; CACHIONI, M. Inclusão digital com tablets entre idosos: metodologia e impacto cognitivo. Psicologia, Saúde \& Doenças, v. 20, n.2, p. 384-401, 2019.

\section{AOTA. AMERICAN OCCUPATIONAL THERAPY ASSOCIATION.}

Occupational therapy practice framework: Domain and process. American Journal of Occupational Therapy, v. 74, n.2 (supl), p. 7412410010p1-7412410010p87, 2020.

BALL, C. et al. The physical-digital divide: Exploring the social gap between digital natives and physical natives. Journal of Applied Gerontology, v. 38, n. 8, p. 1167-1184, 2019.

BORGES, G. R. et al. Um estudo preliminar dos fatores perceptivos subjacentes da preferência dos brasileiros por aparelhos celulares. Perspectivas em Gestão \& Conhecimento, v. 6, n. 1, p. 255-269, 2016.

CALlOW, M. A.; CALLOW, D. D.; SMITH, C. Older adults' intention to socially isolate once COVID-19 stay-at-home orders are replaced with "Safer-at-Home" public health advisories: A survey of respondents in maryland. Journal of Applied Gerontology, v. 39, n.11, p. 1175-1183, 2020.

CASTRO, C. S. S. et al. Distanciamento social durante a pandemia da COVID19, uso de tecnologias e seus impactos para os idosos no Brasil. Population Horizons LARNA Special Issue, p. 1-10, 2020. 
DAVID, M. E.; ROBERTS, J. A. Smartphone Use during the COVID-19 Pandemic: Social Versus Physical Distancing. International Journal of Environmental Research and Public Health, v. 18, n. 3, p. 1-8, 2021.

EYNON, R.; MALMBERG, L. E. Lifelong learning and the Internet: Who benefits most from learning online? British Journal of Educational Technology, v. 52, n. 2, p. 569-583, 2021.

GRÁCIO, R. Museums working with older people in times of pandemic. Working with Older People, v. 24, n. 4, p. 313-319, 2020.

IYENGAR, K. et al. COVID-19 and applications of smartphone technology in the current pandemic. Diabetes \& Metabolic Syndrome: Clinical Research \& Reviews, v. 14, n. 5, p. 733-737, 2020.

KHILNANI, A.; SCHULZ, J.; ROBINSON, L., "The COVID-19 pandemic: new concerns and connections between eHealth and digital inequalities", Journal of Information, Communication and Ethics in Society, v. 18, n. 3, p. 393-403, 2020.

LARSSON-LUND, M.; NYMAN, A. Occupational challenges in a digital society: A discussion inspiring occupational therapy to cross thresholds and embrace possibilities. Scandinavian journal of occupational therapy, v. 27, n. 8, p. 550-553, 2020.

MARTINI, R. Inclusão digital \& inclusão social. Inclusão social, v. 1, n. 1, p. 2123, 2005.

MACHADO, L. R. et al. Mapeamento de competências digitais: a inclusão social dos idosos. ETD-Educação Temática Digital, v. 18, n. 4, p. 903-921, 2016.

MENDES, J. R. et al. Ações educativas em alimentação e nutrição através de tecnologias digitais para um grupo de idosos durante pandemia de Covid-19. Raízes e Rumos, v. 8, n. 1, p. 223-233, 2020.

NUNES, A. L. P. F.; DA CRUZ SILVA, M. B. A extensão universitária no ensino superior e a sociedade. Mal-Estar e Sociedade, v. 4, n. 7, p. 119-133, 2011.

PÁSCOA, G.; GIL, H. Envelhecimento e tecnologia: desafios do século XXI. In: Iberian Conference on Information Systems and Technologies (CISTI). p. 19-22. Coimbra, Portugal. 2019.

RAYMUNDO, T.; GIL, H.; BERNARDO, L. Desenvolvimento de projetos de inclusão digital para idosos. Revista de Estudos Interdisciplinares sobre o Envelhecimento, v. 24, n. 3, p. 22-44, 2019. 
SANDARS, J. et al. Twelve tips for rapidly migrating to online learning during the COVID-19 pandemic. MedEdPublish, v. 9, p. n.1, p. 1-14, 2020.

SEIFERT, A.; COTTEN, S. R.; XIE, B.. A double burden of exclusion? Digital and social exclusion of older adults in times of COVID-19. The Journals of Gerontology: Series B, v. 76, n. 3, p. 99-103, 2021.

TAVARES, M. M. K..; DE SOUZA, S. T. C. Os idosos e as barreiras de acesso às novas tecnologias da informação e comunicação. RENOTE-Revista Novas Tecnologias na Educação, v. 10, n. 1, p. 1-7, 2012.

XIE, B. et al. When Going Digital Becomes a Necessity: Ensuring Older Adults' Needs for Information, Services, and Social Inclusion During COVID-19. Journal of Aging \& Social Policy, v. 32, n. 4-5, p. 460-470, 2020.

Recebido em: 15 de Abril de 2021. Aceito em: 11 de Maio de 2021. 